\title{
INFORMATION TECHNOLOGY IN THE CONSTRUCTION INDUSTRY: MANAGING PROJECTS IN THE AGE OF THE INTERNET
}

\author{
Scott Hudgins and King-Le Chang \\ Ove Arup \& Partners California \\ Harvard University Center for Design Informatics Member \\ scott.hudgins@arup.com
}

\begin{abstract}
- Improved communication
- Team cohesiveness

- Instantaneous information access

- Time savings

- Efficiency

- Improved coordination

- Cost savings
\end{abstract}

Abstract: Information technology in the construction industry opens new frontiers redefining an internet approach to communication and project coordination. The ongoing exploration of such technologies confirms the techniques of transforming the current process of project procurement, design and construction to enable multiple benefits. An internet-based, collaborative information system can offer benefits such as:

The recent Internet technology surge of Project Extranets provides the communication of project information through the instantaneous accessibility of design and construction knowledge. Extranets extend corporate and project information across geographical and organizational boundaries, giving unlimited access to the design and construction team: architects, contractors, consultants and clients. The implementation of knowledge networking through extranet technologies will radically change the way we manage, retrieve, and exchange project information. Ultimately, achieving a significant breakthrough in the construction industry - a new e-approach to design and construction.

Keywords: Project Extranets, Information Technology (IT), New Economy, Communication, Collaboration and Integration.

\section{INTRODUCTION: SYNOPSIS}

As a Research Consultant within Ove Arup \& Partners, I have been an instrumental part of the team in developing new tools that enhance communication within both the delivery team and process itself. Roles have included the initial and current development of a product, liaison with the past, present, and future users in order to develop the brief for the new product and the associated service, and finally all aspects of providing the service to current and future projects.

These tools have been focussed on a Java-based application for use through the World-Wide-Web. Through extensive training, research and self- development I have become proficient in structuring new software applications that apply to our business with special emphasis on creating applications that are conceived from a user's perspective.

These programs are essentially uncomplicated requiring project managers little training to adopt, customize and operate. They also seek to make the best use of current data that is available within project teams, empowering more staff to contribute to the strategic planning and success of the projects themselves.

Personal experience suggests that the returns on investment for both the client and the design teams will be dramatic, both in the hard and soft cost. Clients feel more involved in the process while 
consultants / contractors have data that proves the effectiveness of an extranet.

Recent studies by Harvard University predict that these systems will become, in the very near future, the "hub" of the flow of production information. It is anticipated that all projects will be organized through this approach, becoming as indispensable as CAD has proven in the last ten years.

I therefore outline my philosophy and commitment to this initiative/technology, with the full acknowledgement that others have been instrumental in developing sub-sets of the platform upon which our Project Extranet, integration, has been launched.

\section{IDENTIFICATION OF NEED: SUCCESSFUL PROJECTMANAGEMENT REQUIRES ABSOLUTE VISION.}

In the New Economy, the world of dot.com and cyber space, the Construction Industry has an imponderable dilemma. The business world seeks a more responsive, immediate solution to its opportunities and problems. Virtual organizations are being created through venture capitalist funding that demand immediate, inflated return on their investment. Technology continues to reinvent itself at an astounding rate, typically measured as "net" years.

It is under these pressures that individual Design and Delivery Teams within the construction industry must evolve. Even on medium size projects, it is now understood that the communication systems that are adopted at the start of a project will need to be updated, reconfigured or replaced at least once during the cycle of the delivery program. A project manager cannot assume that the teams will have compatible systems, either in CAD/ CAM / WP or e-mail communication protocols. Configuration Management has become an essential role of the modern project manager. In this new field, it is often the case that experience is the greatest inhibitor to progress.

\subsection{Web-Based Vision}

The absolute vision details features and benefits of the Internet and the effects relative to project management in the construction industry. These benefits can be described in five categories where each can be thought of as a productivity tool providing a means "to enhance quality, quantity and speed of vital information necessary to perform construction tasks. The categories are as follows:

- Resource

- Communication and Network Device

- Marketing Vehicle
- Project Management and Administraion Tool

- Project Collaboration Tool

Many of the web-based, e-commerce processes are new within the industry. Business to business (B2B) relationships cannot be taken for granted and customer to business communication has become much more sophisticated through Partnering, Supply Chain Management and Incentivisation strategies.

\section{OVE ARUP INVESTMENT: INNOVATION.}

Since January 1999, Virtual Construction Group, Arup Computing and Arup Project Management have been investigating, analyzing and developing new systems that can respond to these challenges. Oasys Ltd., a subsidiary of Ove Arup \& Partners, has recently offered the industry a free software package that aims to provide a universal document management tool to the delivery team. Under the banner of Columbus, it unifies and translates, within a central organizer, conflicting consultant and contractor systems, allowing controlled access to project files without reconfiguring individual firms document management systems. By offering it as a free package, we seek to maximize its use as an effective solution to the current malaise of incompatibility of data generating software programs within our industry.

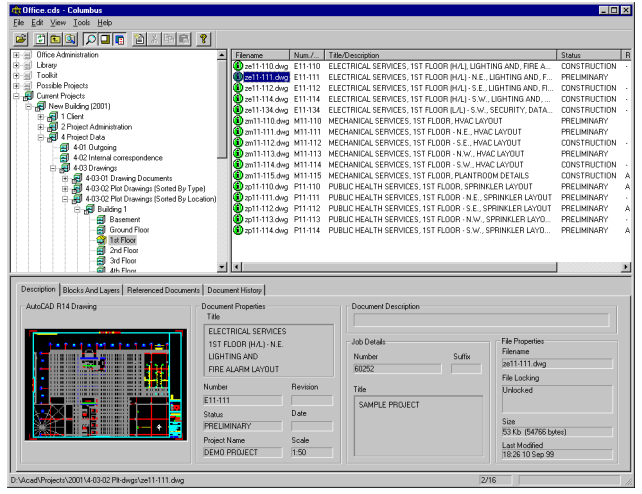

Figure 1. Columbus Document Management Software

Columbus represented Stage 1 of our strategy. Stage 2 is focussed on our day to day ability to communicate, inform, manage and deliver the service in a more effective manner. This is a key role of the project manager and it is crucial in the drive towards more efficient, proactive management of our effort, both in time and resources.

\section{PROJECT EXTRANETS}


Currently the construction industry, which accounts for $\$ 3.4$ trillion in business worldwide, suffers from inefficiency and a high degree of fragmentation. We have recognized that the Internet holds the potential of untangling the "spider web" of collaboration among companies involved in the building process.

Since the early 1990's, Project Extranets have been developed both in the US and in Europe. This tool acts as a virtual office - a hub for all current project data that enabled 24-hour secure access to key project team members. Originally developed in the United States (Blueline Online), these systems have been sponsored through major defense project budgets. Only in the last three years are we seeing that this tool is both practical and affordable throughout our industry.

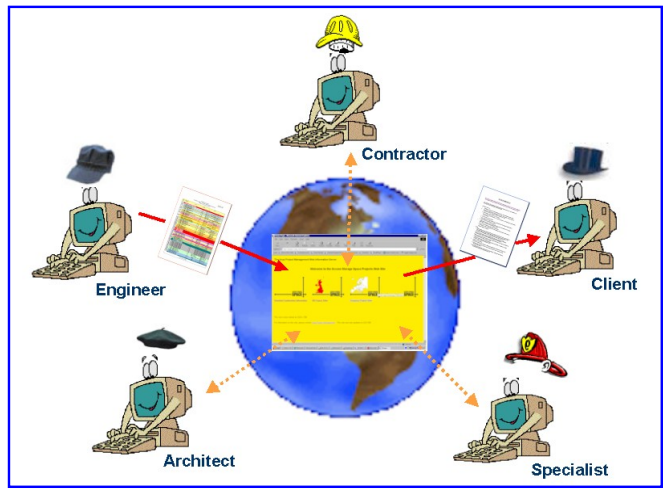

Figure 2. Global Collaboration Diagram

It is also clear that effective use of the Project Extranet will reduce substantially or even eliminate the "paper pushing" element of a Project Manager's job, freeing up time for more valuable and interesting work.

But the current extranet systems focus on the Realization Stage of the project. This is natural, since it is the most intense period for transference of data and the importance of an auditable process for controlling change during construction. However, these systems are overbearing in the early stages of the design and documentation program of any project since they presume:

- A large team on the project

- A high volume of data needing to be transferred

- An elaborate quality assurance procedure that is common to all parties

At the Inception and Design Development Stage of a project, this is often not the case. Even though there may be global teams being formed for largescale projects, the initial team will be small and focused with the client on the brief. Communication must be swift, auditable but flexible to the initial methodology being adopted by the team. The client is often not yet fully committed to the team/scheme or program, but seeks an immediate response to the criteria that has been established.

These teams may only be in operation for a few months, establishing the feasibility of a solution to the brief. Clients are therefore unwilling to make long-term commitments, nor will they sponsor major investment in new hardware/software for the project itself.

It is this focal area of the delivery process that Ove Arup \& Partners developed the web-based extranet system. The brief was based on ensuring a:

- Low cost, but highly functional, package

- Customizable graphics, so that a site could be established quickly and then managed by a client representative (if necessary)

- Highly adaptable format, so that it could be used for both project communication and business to customer (i.e. Marketing) e-commerce tool.

- Inclusive, rather than exclusive, in its compatibility with a large range of software systems. This allows future software systems to be "attached " to Integration to increase its capabilities as the data increases.

- Low maintenance and automatic back-up/ messaging systems included

\section{INTEGRATION DEVELOPMENT}

Arup's policy is to be at the leading edge of our industry and therefore we proposed that a Project Extranets should be used as a communication and document-handling tool for all future projects. Integration, http://www.integration.arup.com is now the vehicle through which the project web-site software and services are being offered directly to our client's and collaborators.

\section{SERVICE OVERVIEW}

An Extranet site streamlines the movement of information between all organizations involved on the project, wherever they may be, enhancing productivity and achieving a significant cost saving through increased access, organization and exchange of project information and reduced requirements for printing and courier services. Team response times are dramatically reduced through e-mail notifications instead of letters/faxes often forgotten at the bottom of in-trays. 
Project Extranets provide easily manageable communication services ideal for projects involving multiple organizations. The main objective of the Project Extranet is to allow the Client and all parties involved in the project to share project specific information, regardless of their location. This provides all team members with easy to locate, up to the minute, project information including drawings, programs and reports preventing the wastage of time and money which results from designing and building to out of date information. All information can be viewed on-line without having to have the application on your PC. E.g. viewing drawings without AutoCAD.

It will also provide all members with a sense of greater involvement and hence ownership which in turn leads to a greater commitment and accountability. Recent projects, which have adopted the Project Extranet template, developed the graphics and successfully used a Project Extranet Site include:

- Greater London Authority Building, London

- Transbay, San Francisco

- Stanhope's Chiswick Park Development, uk

- Intel, Ireland

- Sainsbury's, Egypt

\section{EXTRANET STRUCTURE}

The current Project Extranet comprises the following areas:

- $\quad$ Project and Personal Homepage

- Project Information

General Project Info

Program, Schedule, etc.

Web Links and Addresses

Site Photos / Progress report

- $\quad$ Project Directory

Individual user published contact database.

- $\quad$ Project Diary

Scheduling project events and appointments with automatic email notification.

- Documents

Each member of the project team has the ability to upload and download based on user defined permissions.

- Communications
A server based software application that manages the data, records and follows the sequence of communications - workflow.

Automated Forms: Memorandum / Requests for Information / Submittals / Change Orders

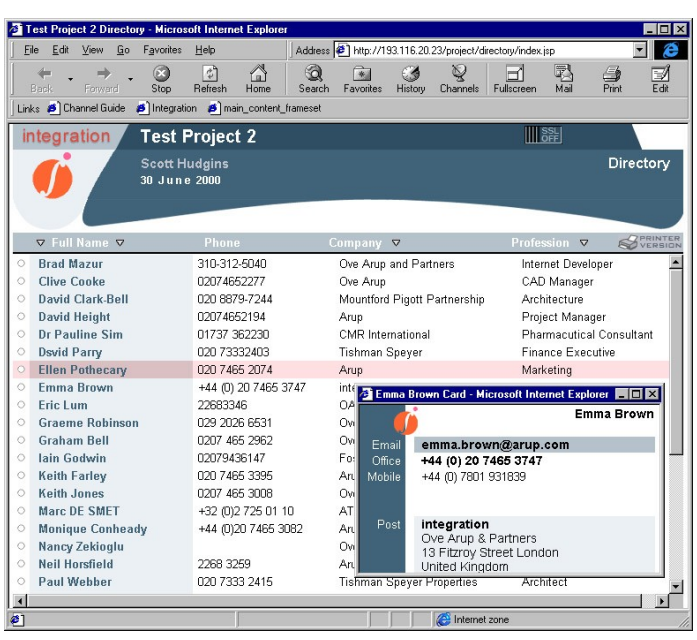

Figure 3. Integration Project Directory

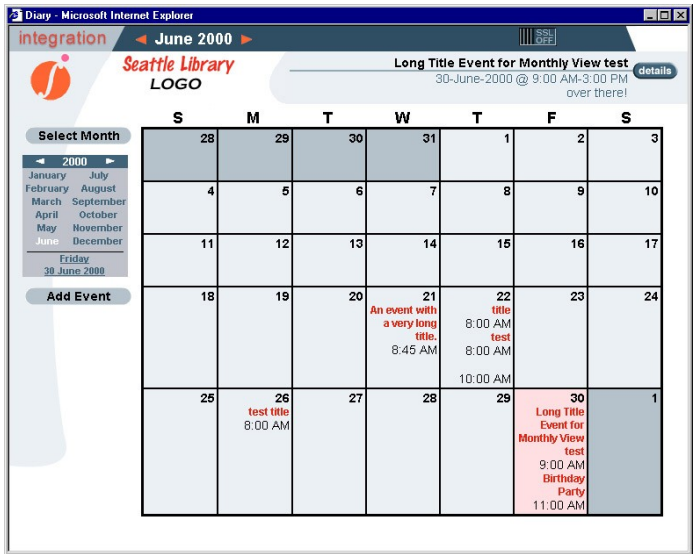

Figure 4. Integration Project Diary 


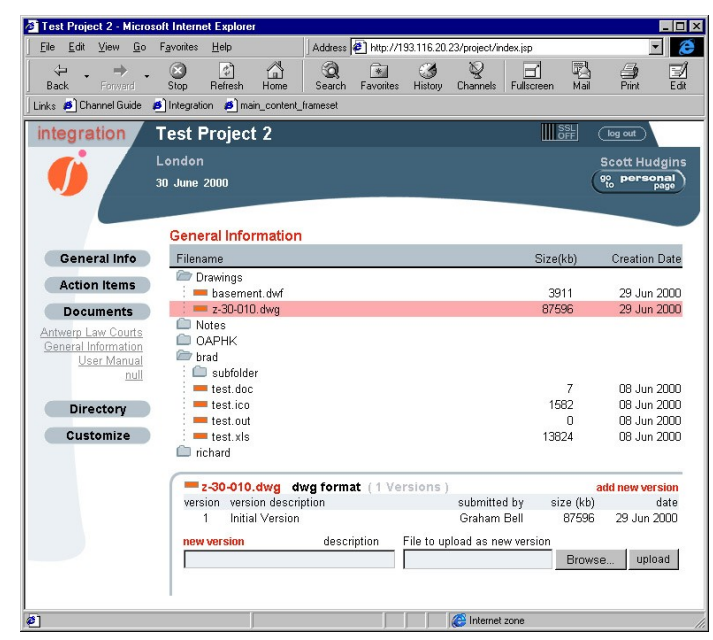

Figure 5. Integration Document Repository

\subsection{Future Developments}

Future developments have been focused on new technologies of internet tools and the global communication infrastructure.

- Fully integrated workflow incorporating program and cost.

- Print Shop facility / mail

- Outlook API: project appointments automatically placed into users outlook account, so user only requires one diary

- Thin Client support - PDA e.g. Palm Pilot, mobile phone (WAP): reduced version of

Project Extranet and / or notification available to these devices.

- Voice over IP: Voice and Data Transfer via the Internet enabling real-time communication. Provides a sense of presence and the potential to hold live meetings via PC.

\section{CONCLUSIONS}

The system that has been developed is now in global operation on over 20 different project sites within the Arup Partnership. The demand is growing significantly at the design stage and is clear that the potential use of Project Extranet systems at the early stages of projects is vital.

The benefits of web-based project management can be summed up in one word - communications. Successful construction is founded in the "effective use of coordination and communication to bring together men, materials and equipment" needed to build the project.

The traditional means of communication will continue to forego a radical change focusing on new tools and methods through devices at remote locations. As in the early days of CAD, many people were reluctant to invest time, energy and commitment to new applications. The benefits are so compelling that the technological investments will secure a place in the New Economy.

\section{Acknowledgments}

The views in this paper are those of the author and have been largely formed during a number of ongoing development projects in Ove Arup \& Partners. The author is grateful for support given by Stuart Cowperthwaite, Sarah Bowden, Paul RichardWebber, Brad Rendle, King-Le Chang, Richard Bussell and the principal sponsor of this work Mark Bostock.

\section{REFERENCES}

[1] Cowperthwaite, S., "Information Sharing in Building - Project Modelling and Extranets", CIBSE National Conference, Harrogate International Centre, 3 October 1998.

[2] 2000 Edition The Guide to E-Commerce and Web-Based Project Management in Construction, WPL Publishing Company., 2000.

[3] Extranet World, http://www.extranets.cc

[4] integration, http://www.integration.arup.com

[5] Columbus, http://www.columbus.arup.com 